\title{
Cerebral oxygen delivery in newborns with congenital heart disease by phase contrast MRI
}

\author{
Jessie Mei Lim*, Theo Kingdom, Brahmdeep Saini, Susan Blaser, Lars Grosse-Wortmann, Shi-Joon Yoo, \\ Edward J Hickey, Christopher Macgowan, Steven Miller, Mike Seed \\ From 18th Annual SCMR Scientific Sessions \\ Nice, France. 4-7 February 2015
}

\section{Background}

Congenital heart disease (CHD) is associated with in utero brain dysmaturation and abnormal cerebral vasculature [1,2]. Phase contrast (PC) MRI allows non-invasive quantification of cerebral blood flow (CBF) and, combined with arterial oxygen saturation $\left(\mathrm{SaO}_{2}\right)$ and haemoglobin $(\mathrm{Hgb})$ concentration, can measure cerebral oxygen delivery $\left(\mathrm{CDO}_{2}\right)$. We hypothesized that $\mathrm{CDO}_{2}$ would be lower in newborns with $\mathrm{CHD}$ than normals.

\section{Methods}

We measured CBF and brain volume (BV) in 32 unsedated newborns (21 normals and $11 \mathrm{CHD}$ ) as part of an IRB approved study using a 1.5T Siemens Avanto MRI. CBF was measured according to a previously published technique using a single PC acquisition prescribed perpendicular to both internal carotid arteries (ICA) and basilar artery (BA) at the clivus level [3]. We also measured flows in the vertebral arteries (VA), common carotid arteries (CCA) and internal jugular veins (IJV) using a PC acquisition through the neck. Flows were quantified and indexed to $\mathrm{BV}$ calculated from a high resolution $3 \mathrm{D}$ T2W FSE acquisition. $\mathrm{SaO}_{2}$ and $\mathrm{Hgb}$ concentrations were measured using conventional blood gases in order to calculate: $\mathrm{CDO}_{2}=\mathrm{SaO}_{2} \times[\mathrm{Hgb}] \times 1.36 \times \mathrm{CBF}$ [4]. We compared net and indexed $\mathrm{CBF}$ and $\mathrm{CDO}_{2}$ in the two groups with an unpaired $t$-test with Welch's correction (Table 1). An analysis of CHD subgroups was also performed. The relationship between $\mathrm{CBF}$ and gestational age (GA) and agreement between BA vs VA flow sum was examined using Pearson's R.

\section{Results}

Subjects were scanned at a mean corrected GA of 40 weeks. MRI was performed prior to cardiac surgery in all CHD subjects. The two groups had no difference in GA $(p=0.07)$. There were trends towards lower mean net and indexed $\mathrm{CBF}$ in $\mathrm{CHD}$ newborns compared with controls (control net CBF $138 \pm 14$ vs CHD $118 \pm 10 \mathrm{ml} / \mathrm{min}$ ( $\mathrm{p}=0.26$ ), control indexed CBF $0.341 \pm 0.027$ vs CHD 0.321 $\pm 0.020 \mathrm{ml} / \mathrm{min} / \mathrm{ml}_{\mathrm{BV}}(\mathrm{p}=0.56)$ ) (Table 1, Figure 1a,b). A similar trend in mean net and indexed $\mathrm{CDO}_{2}$ was observed in CHD newborns compared with controls (control net $\mathrm{CDO}_{2} 2749 \pm 284$ vs CHD $2191 \pm 159 \mathrm{ml}_{\mathrm{O} 2} / \mathrm{min}(\mathrm{p}=0.1)$, control indexed $\mathrm{CDO}_{2} 6.81 \pm 0.54$ vs $\mathrm{CHD} 5.99 \pm 0.31 \mathrm{ml}_{\mathrm{O} 2} /$ $\left.\mathrm{min} / \mathrm{ml}_{\mathrm{BV}}(\mathrm{p}=0.19)\right)$ (Figure 1c,d). In keeping with one previous study (3), CBF increased with $\mathrm{GA}(\mathrm{r}=0.7, \mathrm{p}=<0.0001)$. The VA and BA flows were highly correlated $(r=0.8$, $\mathrm{p}=0.0002$ ), indicating good accuracy of the $\mathrm{PC}$ assessment of CBF. There were no significant differences in measured parameters between subgroups of CHD.

\section{Conclusions}

Although the differences between $\mathrm{CBF}$ and $\mathrm{CDO}_{2}$ in this small sample of subjects were not statistically significant, there was a clear trend towards lower values for both parameters in CHD subjects. We propose that with a larger sample size, these differences will be significant, in keeping with a demonstrable relationship between cerebral oxygenation and brain maturation in CHD fetuses and newborns.

\section{Funding}

N/A.

The Hospital for Sick Children, Mississauga, ON, Canada 
Table 1 Table of mean values for all parameters measured, listed as mean \pm SEM. P-values refer to comparisons of each subject group to the control group. The CHD subgroups are as follows: single ventricle physiology (SV), transposition of the great arteries (TGA), coarctation of the aorta (CoA), cyanotic and non-cyanotic CHD.

\begin{tabular}{|c|c|c|c|c|c|c|c|c|c|c|c|}
\hline \multirow[b]{2}{*}{$\begin{array}{l}\text { Patient } \\
\text { Type }\end{array}$} & \multirow[b]{2}{*}{$\begin{array}{c}\text { Brain } \\
\text { Volume } \\
(\mathrm{ml})\end{array}$} & \multirow[b]{2}{*}{$\mathrm{SaO}_{2}(\%)$} & \multirow[b]{2}{*}{$\mathrm{Hgb}(\mathrm{g} / \mathrm{dL})$} & \multicolumn{3}{|c|}{ Net Value } & \multicolumn{5}{|c|}{ Indexed to Brain Volume } \\
\hline & & & & $\begin{array}{c}\text { CBF (ml/ } \\
\text { min) }\end{array}$ & $\begin{array}{l}\text { P-Value } \\
\text { for CBF }\end{array}$ & $\begin{array}{c}\mathrm{CDO}_{2} \\
\left(\mathrm{ml}_{\mathrm{O} 2} / \mathrm{min}\right)\end{array}$ & $\begin{array}{l}\text { P-Value } \\
\text { for } \mathrm{CDO}_{2}\end{array}$ & $\begin{array}{c}\text { CBF } \\
(\mathrm{ml} / \mathrm{min} / \\
\left.\mathrm{ml}_{\mathrm{BV}}\right)\end{array}$ & $\begin{array}{l}\text { P-Value } \\
\text { for CBF }\end{array}$ & $\begin{array}{c}\mathrm{CDO}_{2}\left(\mathrm{ml}_{\mathrm{O} 2} /\right. \\
\left.\mathrm{min} / \mathrm{ml}_{\mathrm{BV}}\right)\end{array}$ & $\begin{array}{l}\text { P-Value } \\
\text { for } \mathrm{CDO}_{2}\end{array}$ \\
\hline $\begin{array}{c}\text { Normal } \\
(n=21)\end{array}$ & $395 \pm 12$ & 98 & 15 & $138 \pm 14$ & & $2749 \pm 284$ & & $\begin{array}{c}0.341 \pm \\
0.027\end{array}$ & & $6.81 \pm 0.54$ & \\
\hline $\begin{array}{c}\text { CHD } \\
(n=11)\end{array}$ & $364 \pm 14$ & $90 \pm 2.9$ & $15.4 \pm 0.44$ & $118 \pm 10$ & 0.26 & $2191 \pm 159$ & 0.10 & $\begin{array}{c}0.321 \pm \\
0.020\end{array}$ & 0.56 & $5.99 \pm 0.31$ & 0.19 \\
\hline SV $(n=4)$ & $364 \pm 31$ & $85 \pm 6.4$ & $15.5 \pm 1.1$ & $122 \pm 22$ & 0.58 & $2101 \pm 299$ & 0.15 & $\begin{array}{c}0.328 \pm \\
0.036\end{array}$ & 0.78 & $5.69 \pm 0.49$ & 0.15 \\
\hline TGA $(n=4)$ & $347 \pm 13$ & $89 \pm 1.5$ & $15.1 \pm 0.38$ & $113 \pm 13$ & 0.23 & $2058 \pm 199$ & 0.06 & $\begin{array}{c}0.329 \pm \\
0.041\end{array}$ & 0.82 & $5.97 \pm 0.63$ & 0.34 \\
\hline $\operatorname{CoA}(n=3)$ & $385 \pm 28$ & $\begin{array}{l}99 \pm \\
0.67\end{array}$ & $15.8 \pm 0.73$ & $117 \pm 20$ & 0.46 & $2487 \pm 382$ & 0.61 & $\begin{array}{c}0.301 \pm \\
0.032\end{array}$ & 0.38 & $6.40 \pm 0.59$ & 0.63 \\
\hline $\begin{array}{c}\text { Cyanotic } \\
(n=7)\end{array}$ & $365 \pm 14$ & $85 \pm 2.9$ & $15.7 \pm 0.46$ & $124 \pm 12$ & 0.47 & $2198 \pm 135$ & 0.09 & $\begin{array}{c}0.339 \pm \\
0.027\end{array}$ & 0.96 & $6.04 \pm 0.36$ & 0.24 \\
\hline $\begin{array}{c}\text { Non- } \\
\text { Cyanotic } \\
(n=4)\end{array}$ & $361 \pm 31$ & $\begin{array}{c}100 \pm \\
0.5\end{array}$ & $15.0 \pm 0.98$ & $106 \pm 18$ & 0.22 & $2177 \pm 411$ & 0.29 & $\begin{array}{c}0.290 \pm \\
0.025\end{array}$ & 0.19 & $5.89 \pm 0.66$ & 0.31 \\
\hline
\end{tabular}

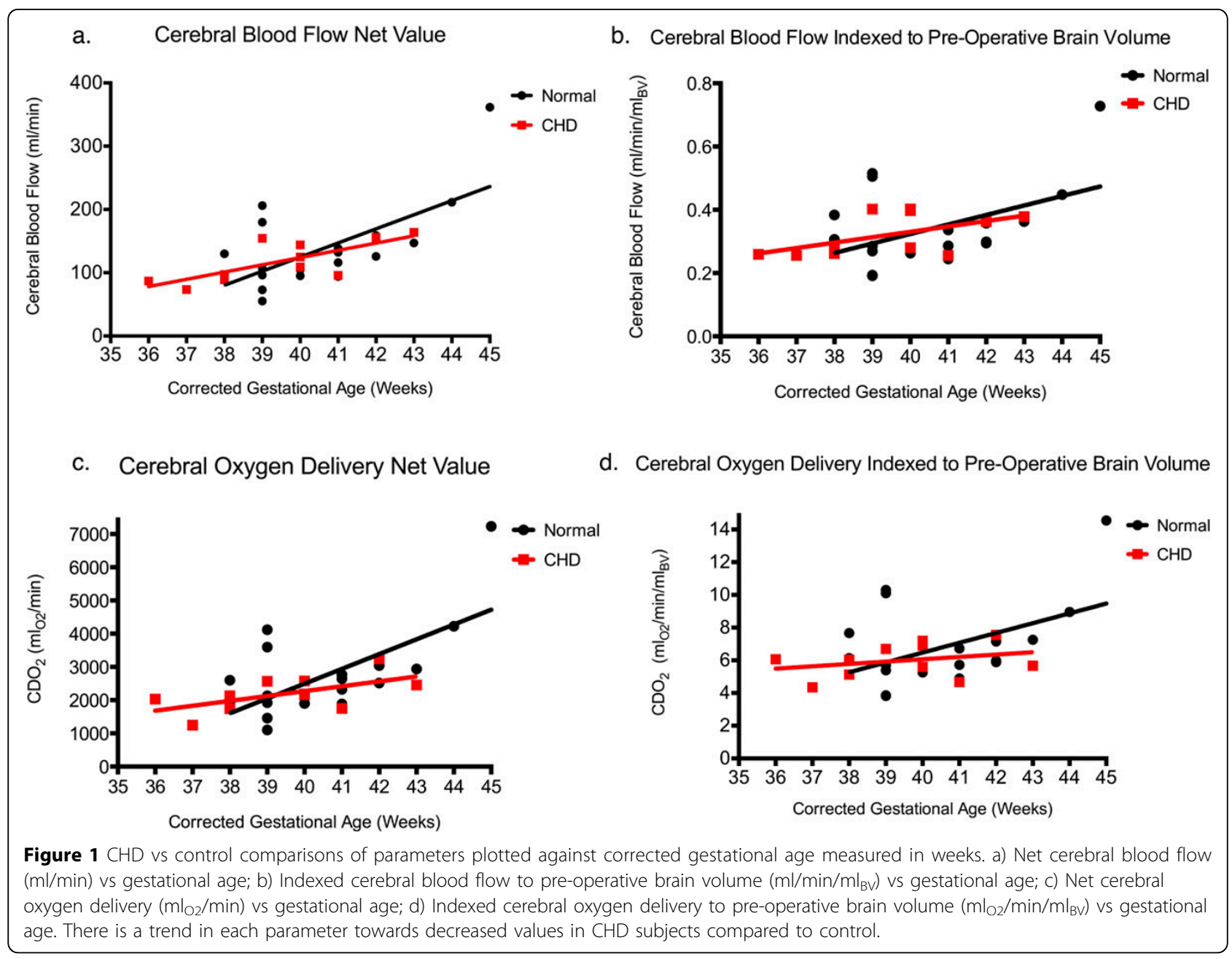


Published: 3 February 2015

\section{References}

1. Miller S, et al: N Eng N Med 2007.

2. Kinnear C, et al: Circulation 2008.

3. Varela M, et al: NMR in Biomedicine 2012.

4. Rudolph AM: Congenital Diseases of the Heart Wiley; 2009.

doi:10.1186/1532-429X-17-S1-M9

Cite this article as: Lim et al:: Cerebral oxygen delivery in newborns with congenital heart disease by phase contrast MRI. Journal of Cardiovascular Magnetic Resonance 2015 17(Suppl 1):M9.

Submit your next manuscript to BioMed Central and take full advantage of:

- Convenient online submission

- Thorough peer review

- No space constraints or color figure charges

- Immediate publication on acceptance

- Inclusion in PubMed, CAS, Scopus and Google Scholar

- Research which is freely available for redistribution

Submit your manuscript at www.biomedcentral.com/submit
Ciomed Central 